\title{
Cloning and Functional Characterization of Key Enzymes in Putative Octadecanoid Pathway of Physcomitrella patens
}

\author{
P.K.G.S.S. Bandara ${ }^{*}$, R.P. Karunagoda ${ }^{1}$, K. Takahashi and K. Nabeta \\ Division of Applied Bioscience, Research Faculty of Agriculture \\ Hokkaido University, Sapporo \\ Japan
}

\begin{abstract}
Jasmonic acid and its metabolites are ubiquitously occurring lipid-derived signaling compounds that regulate growth, development and defense processes in flowering plants. However, their functions in lower land plants have not been well characterized yet. The model moss, Physcomitrella patens is a member of bryophytes and represents a key evolutionary position between green algae and flowering plants. According to the proposed land plant evolution, jasmonic acid signaling pathway has evolved after the evolutionary split of bryophytes and vascular plants, indicating its absence in bryophytes. However, the putative key homologous genes involved in jasmonic acid biosynthesis and its signaling

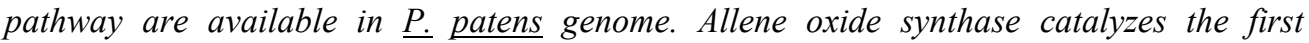
committed step of jasmonic acid biosynthesis in flowering plants. A putative allene oxide synthase gene of $\underline{P}$. patens was cloned and shown to have the same in vitro function as observed in flowering plants. Moreover, based on substrate specificity, plant 12-oxophytodienoic acid reductases can be classified into two groups, group I and group II, of which only group II isozymes involve in jasmonic acid biosynthesis. Six putative 12-oxophytodienoic acid reductase genes, among which only one represents group II, were identified in $\underline{P}$. patens genome. Two 12-oxo-phytodienoic acid reductase genes, which represent group I and group II, were cloned and characterized. Striking results were obtained since the 12-oxo-phytodienoic acid reductase, which represents group II, exhibited group I type activity. This unusual functional property clearly explains why $\underline{P}$. patens, probably all the bryophytes, does not contain jasmonic and its signaling pathway though it contains the corresponding homologous genes. Moreover, the ancestral plant 12-oxophytodienoic acid reductases might have possessed group I type activity and early precursors of jasmonic acid might have functioned as signaling molecules in ancestral land plants instead of jasmonic acid.
\end{abstract}

Key words: Jasmonic acid, Physcomitrella patens, bryophytes, Allene oxide synthase

\section{INTRODUCTION}

Jasmonic acid (JA) and its metabolites, collectively known as jasmonates, are plant hormones that mediate plant stress responses and development processes (Wasternack et al., 1998). Among JAs, (+)-7-iso-Jasmonoyl-L- isoleucine (JA-Ile) is the most bioactive endogenous jasmonate (Fonseca et al., 2008). JA is biosynthesized through the octadecanoid

\footnotetext{
Department of Agricultural Biology, Faculty of Agriculture, University of Peradeniya, Peradeniya, Sri Lanka Author for correspondence: sarathbandara2003@yahoo.co.uk
} 
pathway initiated with the oxygenation of $\alpha$-linolenic acid by 13-lipoxygenase (13-LOX) to generate 13(S)-hydroperoxy-(9Z,11E,15Z)-octadecatrienoic acid (13(S)-HPOT) and it is then converted into unstable $(12,13 S)$-epoxy-(9Z, $11 E, 15 Z)$-octadecatrienoic acid $(12,13 S$-EOT) by allene oxide synthase (AOS) followed by the conversion into 12-oxophytodienoic acid (OPDA) by allene oxide cyclase (AOC). In the absence of AOC, unstable 12,13-EOT is spontaneously hydrolyzed into racemic OPDA, $\alpha$-ketol and $\gamma$-ketol (Schaller et al., 2005). The OPDA is then localized to peroxisome and subsequently reduced by group II 12oxophytodienoic acid reductase (group II OPR) to yield 3-oxo-2-(2'-(Z)-pentenyl)cyclopentane-1-octanoic acid (OPC-8:0) (Breithaupt et al., 2009). Based on substrate specificity conferred by two gate keeping amino acid residues located in active site cavity of OPRs, plant OPRs can be classified into two groups, group I and group II OPRs. Group I OPRs, whose gate keeping residues are tyrosines, can reduce only (-)-cis-OPDA into (-)-cisOPC-8:0, which is not a precursor of JA. In contrast, the corresponding positions of group II OPRs are occupied by relatively smaller phenylalanine and histidine residues, forming a larger active site cavity that accept both (-)-cic-OPDA and (+)-cis-OPDA, which is a precursor of JA (Breithaupt et al., 2009). Finally, Three steps of $\beta$-oxidation are required for shortening the octanoic acid side chain of the (+)-cis-OPC-8:0 to biosynthesis iso-JA which is simultaneously isomerized to JA (Fig. 1) (Schaller et al., 2005).
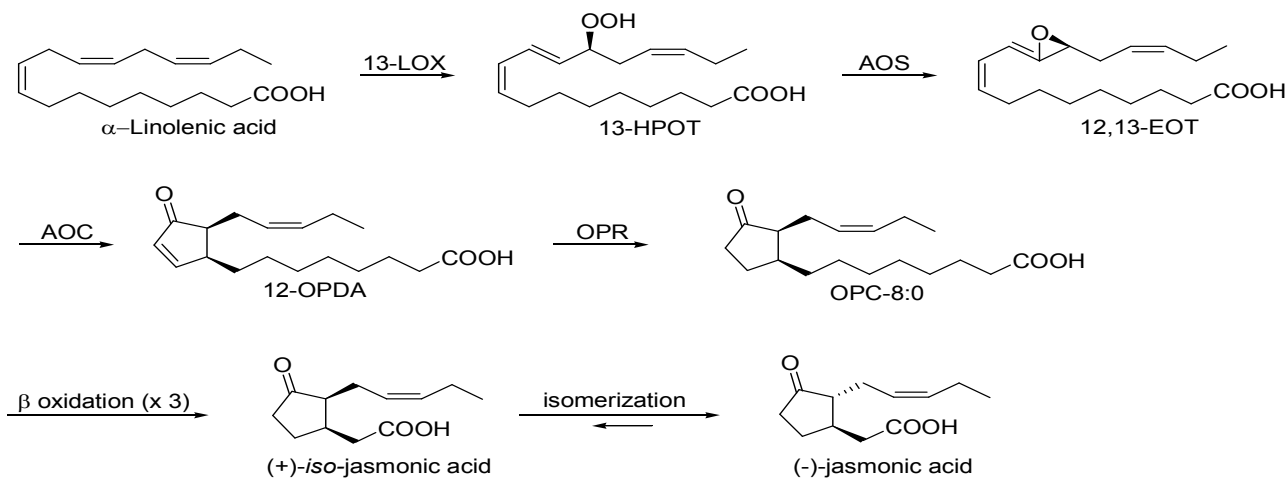

Fig. 1. The octadecanoid pathway

Recently, the entire picture of JAs perception in flowering plants has been revealed (Santner \& Estelle, 2009). The transcriptional repressor of jasmonate responsive genes (JAZ1) is targeted by $\mathrm{SCF}^{\mathrm{COI1}}$ E3 ubiquitin ligase complex for degradation via the $26 \mathrm{~S}$ proteasome, releasing the transcriptional factor (MYC2) of jasmonate responsive genes (Chini et al., 2007). The interaction of JAZ1 with SCF ${ }^{\mathrm{COII}}$ is enabled by JA-Ile (Fonseca et al., 2008). However, little information is available about the JA biosynthesis and its function in lower land plants.

The model moss Physcomitrella patens belongs to phylum Bryophyta and has diverged from vascular plants by about 400 million years ago (Rensing et al., 2008). P. patens is an ideal model for genetic studies because it is the only plant known to have the highest homologous recombination ability enabling easy gene targeting and its haploid dominant life cycle makes genetic studies easier (Cove, 2005). The genome sequencing of $P$. patens was recently completed (Rensing et al., 2008). According to the proposed land plant evolution, JA signaling pathway has evolved after the evolutionary split of bryophytes and vascular plants, indicating its absence in bryophytes (Rensing et al., 2008). However, the presence of other well known plant hormone signaling pathways such as, cytokinin (Schwartzenberg et al., 
2007), abscisic acid (Komatsu et al., 2009) and auxin (Muller et al., 2009) has been proven in P. patens.

So far, there are no clear explanations as to why P. patens (probably all the bryophytes) does not contain JA or its signaling pathway even though its genome harbors the key homologous genes for both biosynthetic and signaling pathways of JA. Here we describe the unusual function of PpOPR3 which causes a failure of JA biosynthesis in P. patens.

\section{MATERIALS AND METHODS}

\section{Plant Materials}

P. patens was cultured on BCDTAG agar medium under continuous white fluorescence light at $25^{\circ} \mathrm{C}$, as previously described (Nishiyama et al., 2000).

\section{Cloning of PpAOS1, PpOPR1, and PpOPR3 Genes}

Total RNA was extracted from 3 week-old P. patens and reverse transcription (M-MLV Reverse Transcriptase, Invitrogen, Carlsbad, CA, USA) was performed according to the manufacturer's instructions to yield cDNA. Using cosmoss genomic database (http://www.cosmoss.org/), two PpAOSs and six PpOPRs genes were identified. Amino acid sequence alignment was performed using ClustalW multiple sequence alignment program (http://clustalw.ddbj.nig.ac.jp/top-e.html). Chloroplast or peroxisomal transit signal peptides were analyzed by iPSORT program (http://ipsort.hgc.jp/) and PSORT program (http://psort.hgc.jp/form.html), respectively. Molecular masses and PI values of recombinant proteins were predicted by ExPASy proteomics server (http://au.expasy.org/tools/pi tool.html). To amplify the open reading frames of PpAOS1 (XP_001767870), PpOPR1 (XP_001751301) and PpOPR3 (Pp1s62_139V6.1), the following primers with restriction cutting sites were used. The restriction sites of primers were highlighted by bold letters.

For PpAOS1; forward primer with Nde1 site (PpAOS1F: CTTCATATGGCAGTCCCTTCATCCAAG) and reverse primer with Xho1 site (PpAOS1R: GGAACTCGAGCTTTTTGAGATGGAAA), for PpOPR1; forward primer with Nde1 site (PpOPR1F: TTCATATGGATTGTTACTGGTCTTATGGC) and reverse primer with Sal1 site (PpOPR1R: AAGTCGACAGCCTGGCATGCTGTCT), and for PpOPR3; forward primer with Sac1 site (PpOPR3F: ATGAGCTCAATTATACAGCCATTTCC) and reverse primer with Pst 1 site (PpOPR3R: ATCTGCAGTCATACATTCTCAACCTGAA).

The PCR reaction mixture (total volume $50 \mu \mathrm{l})$ consists of $10 \mu \mathrm{l}$ of dNTP mixture $(2.0 \mathrm{mM}$ each dNTP), $1 \mu \mathrm{l}$ from each primer $(10 \mu \mathrm{M}), 0.5 \mu \mathrm{l}$ of cDNA, $25 \mu \mathrm{l}$ of KODFX PCR buffer ( $2 \mathrm{X}$ ), $0.5 \mu \mathrm{l}$ of KOD FX DNA polymerase (Toyobo, Japan) and $12 \mu \mathrm{l}$ of Milli-Q water. The PCR thermal cycle was $94^{\circ} \mathrm{C}$ for 2 min (initial denaturation), $98{ }^{\circ} \mathrm{C}$ for $10 \mathrm{~s}, 58^{\circ} \mathrm{C}$ for $30 \mathrm{~s}$, and $68{ }^{\circ} \mathrm{C}$ for $1 \mathrm{~min}$ and $30 \mathrm{~s}\left(40\right.$ cycles), and $68{ }^{\circ} \mathrm{C}$ for $10 \mathrm{~min}$ (final extension). The amplified sequences of PPAOS1, PpOPR1 and PPOPR3 were analyzed by agarose gel electrophoresis. The PCR products were excised from gels and ligated into pBluescript II SK $(+)$ vector (Stratagene, USA) for sequencing. Sequencing was carried out by dideoxy chain termination method. Sequencing PCR was performed with BigDye ${ }^{\circledR}$ Terminator V 3.1 cycle 
sequencing kit (AB Applied Biosystems, USA). After sequence verification of all the three genes, plasmids (SK-PpAOS1, SK-PpOPR1 and SK-PpOPR3) were digested with corresponding restriction enzymes (Takara, Otsu, Japan). The digested contents were separated on agarose gel and ligated into the protein expression vectors digested with corresponding enzymes. For PpAOS1 and PpOPR1, pET23a $(+)$ expression vector (Novagen, USA) was used, whereas, for PpOPR3, pQE30 (Qiagen, USA) expression vector was used. Expression constructs were transformed into $E$. coli strains BL-21(DE3) and M15 for recombinant protein synthesis. E. coli colonies harboring expression vectors were incubated in LB medium containing ampicillin $(100 \mu \mathrm{g} / \mathrm{ml})$ at $25{ }^{\circ} \mathrm{C}$ by shaking at $200 \mathrm{rpm}$. After the cultures reached $\mathrm{OD}_{600}$ of $0.8-1.0$, the recombinant protein synthesis was induced by addition of IPTG (Final concentration of $0.2 \mathrm{mM}$ ). After incubation for $4 \mathrm{~h}$, the cells were collected by centrifugation at $7000 \mathrm{~g}$ for $20 \mathrm{~min}$ at $4{ }^{\circ} \mathrm{C}$ and washed with $50 \mathrm{mM}$ sodium phosphate buffer ( $\mathrm{pH}$ 7.4). The cells were resuspended in $50 \mathrm{mM}$ sodium phosphate buffer (pH 7.4) containing $0.3 \mathrm{M} \mathrm{NaCl}$ and $1 \mathrm{mM}$ PMSF and ruptured by ultrasonication. The cell debris was removed by centrifugation at $14000 \mathrm{~g}$ for $20 \mathrm{~min}$. The recombinant proteins with polyhistidine tag were purified by Ni-NTA agarose column chromatography $(2 \mathrm{ml}$, GE healthcare, USA) for activity analysis.

\section{Activity Analysis of Recombinant PpAOS1}

For the analysis of recombinant PpAOS1 activity, $6 \mu \mathrm{g}$ of 13(S)-HPOT was reacted with 2 $\mu \mathrm{g}$ of purified recombinant PpAOS1 protein in a total volume of $0.1 \mathrm{ml}$ of $50 \mathrm{mM}$ sodium phosphate buffer (pH 6.0) at $25^{\circ} \mathrm{C}$ for $45 \mathrm{~min}$. The products were enolized with $0.2 \mathrm{ml}$ of 0.1 $\mathrm{M} \mathrm{KOH}$ at $25^{\circ} \mathrm{C}$ for $1 \mathrm{~h}$ to convert racemic cis-OPDA into racemic trans-OPDA (since the GC-MS column used in this study can separate trans isomers more easily than cis isomers) and methylated by ethereal diazomethane for chiral GC-MS analysis by GC-MS spectrometer (1200L/Varian CP 3800, USA) with chiral column $\beta$-DEX 120 (30 m x 0.25 $\mathrm{mm}$ x $0.25 \mu \mathrm{m}$; Supelco, USA).

\section{Activity Analysis of Recombinant PpOPR1 and PpOPR3}

The same reaction as described above was followed together with recombinant PpAOS1 to provide the racemic cis-OPDA, the substrate of PpOPRs. Activity analysis was performed in $0.1 \mathrm{ml}$ of $50 \mathrm{mM}$ sodium phosphate buffer ( $\mathrm{pH} 7.0$ ) consisting of $6 \mu \mathrm{g}$ of 13(S)-HPOT, 1 mM NADPH, $2 \mu \mathrm{g}$ of recombinant PpAOS1 and $1 \mu \mathrm{g}$ of recombinant PpOPR1 or PpOPR3 at $25{ }^{\circ} \mathrm{C}$ for $45 \mathrm{~min}$. Enolized and methylated products were analyzed by chiral GC-MS as described above.

\section{RESULTS AND DISCUSSION}

In jasmonic acid biosynthetic pathway, AOS catalyzes the first committed step by which 13(S)-HPOT is converted into unstable allene oxide. To understand the activity of PpAOS1 toward 13(S)-HOPT, in vitro reaction was performed with 13(S)-HPOT. Since unstable $12,13 S$-EOT is spontaneously converted into racemic OPDA, $\alpha$-ketol, and $\gamma$-ketol, AOS enzyme activity can be determined by analyzing the presence of the above compounds. As expected, the presence of racemic OPDA was observed by chiral GC-MS analysis (Fig. 2). Peaks of methylated (+)-trans-OPDA and methylated (-)-trans-OPDA whose retention times were $87 \mathrm{~min}$ and $89 \mathrm{~min}$, respectively, were identified on chiral GC-MS profile (Fig. 2). 


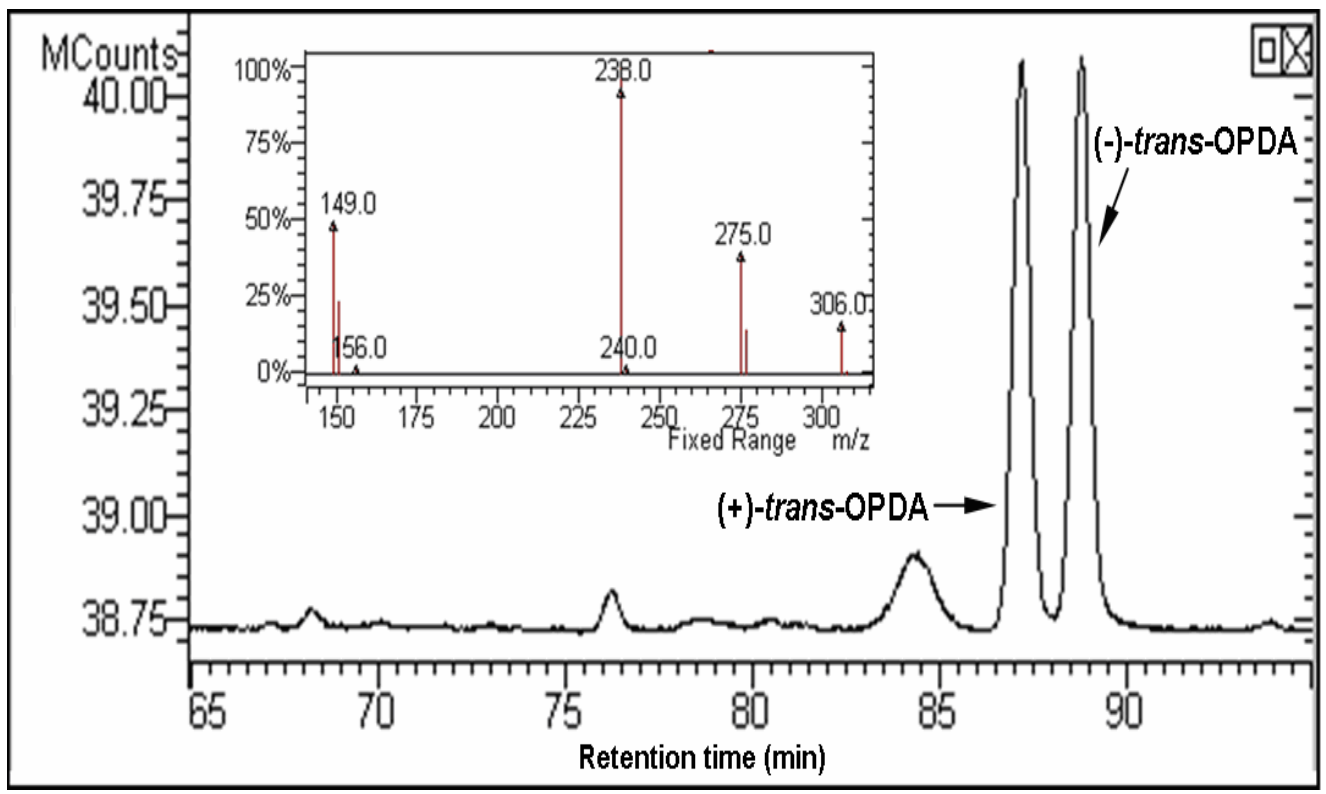

Fig. 2. Chiral GC-MS profile of methylated racemic OPDA from the reaction of recombinant PpAOS1 with 13(S)-HPOT. Inset indicates the mass spectra of methylated OPDA

An ion peak at $m / z 306$ which corresponds to the molecular ion peak [M] $]^{+}$of OPDA methyl ester was observed together with unique fragment ion peaks at $m / z 275\left(\left[\mathrm{M}-\mathrm{OCH}_{3}\right]^{+}\right), \mathrm{m} / \mathrm{z}$ $238\left(\left[\mathrm{M}^{-} \mathrm{C}_{5} \mathrm{H}_{9}\right]^{+}\right)$, and $m / z 149\left(\left[\mathrm{M}-\mathrm{C}_{9} \mathrm{H}_{17} \mathrm{O}_{2}\right]^{+}\right)$(Fig. 2). 13-LOX activity of some P. patens LOXs (Anterola et al., 2009) and AOC activity of P. patens AOCs (Hashimoto et al., 2011) have already been reported. Together with our results, it is clear that $P$. patens has conserved the first half of octadecanoid pathway which takes place in the chloroplast. Then, $(+)$-cisOPDA generated by the first half of octadecanoid pathway in chloroplast should be translocated into peroxisome for the initiation of the second half. In peroxisome, group II OPRs convert (+)-cis-OPDA into (+)-cis-OPC-8:0, a precursor of JA. Amino acid sequence alignment of PpOPRs with those of flowering plant OPRs revealed that PpOPR3 is the only possible candidate of being a group II OPR in P. patens (Fig. 3). AtOPR3, SIOPR3, OsOPR7 and ZmOPR7 are already known group II OPRs whereas, AtOPR1, AtOPR2, OsOPR1 and ZmOPR5 are known group I OPRS which reduce only (-)-cis-OPDA that is not a precursor of JA. As described above, group II OPRs have conserved phenylalanine and histidine residues at substrate specificity determining positions (indicated by arrows) conferring relax substrate specificity to group II OPRs to accept both (+)- and (-)-cis-OPDA (Fig. 3). 


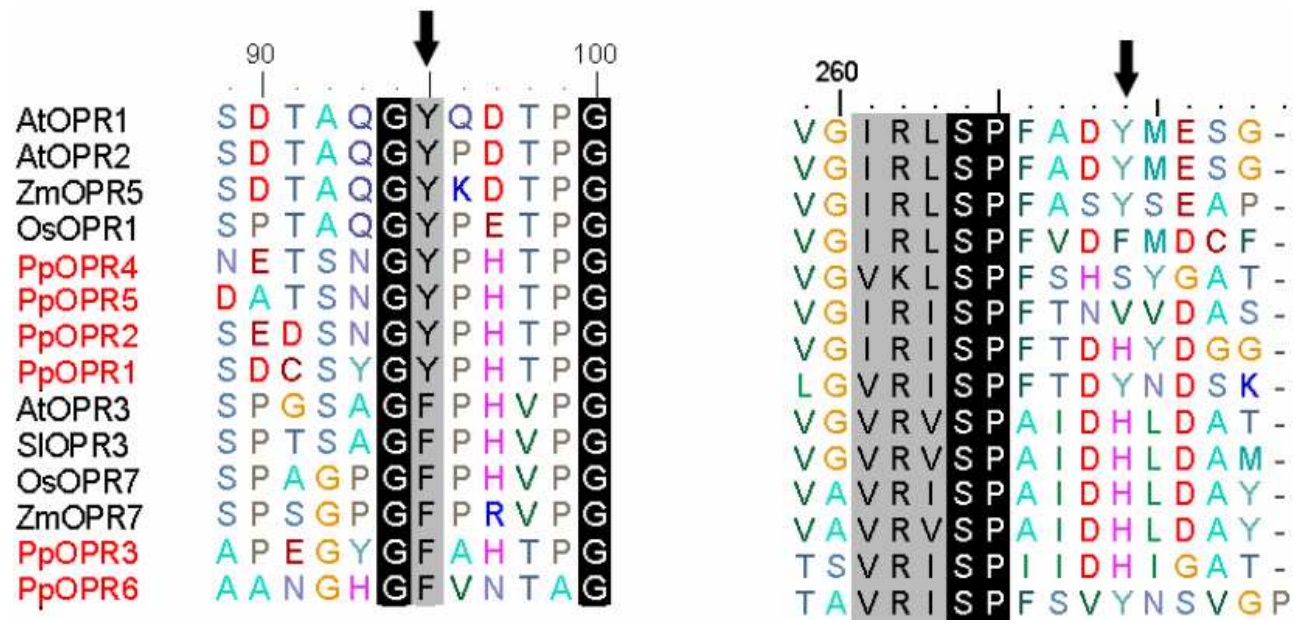

Fig. 3. Two portions of plant OPRs amino acid sequence alignment. Arrows indicate the two gate keeping amino acid residues which confer the substrate specificity to OPRs. Arabidopsis thaliana (AtOPR), Solanum lycopersicum (SlOPR), Oryza sativa (OsOPR), Zea mays (ZmOPR), Physcomitrella patens (PpOPR)

PpOPR1 contains two tyrosine residues at substrate specificity determining positions (Fig. 3 ), and therefore, it should have group I type activity. As expected PpOPR1 preferentially reduces the (-)-cis-OPDA into (-)-cis OPC-8:0 (Fig. 4).

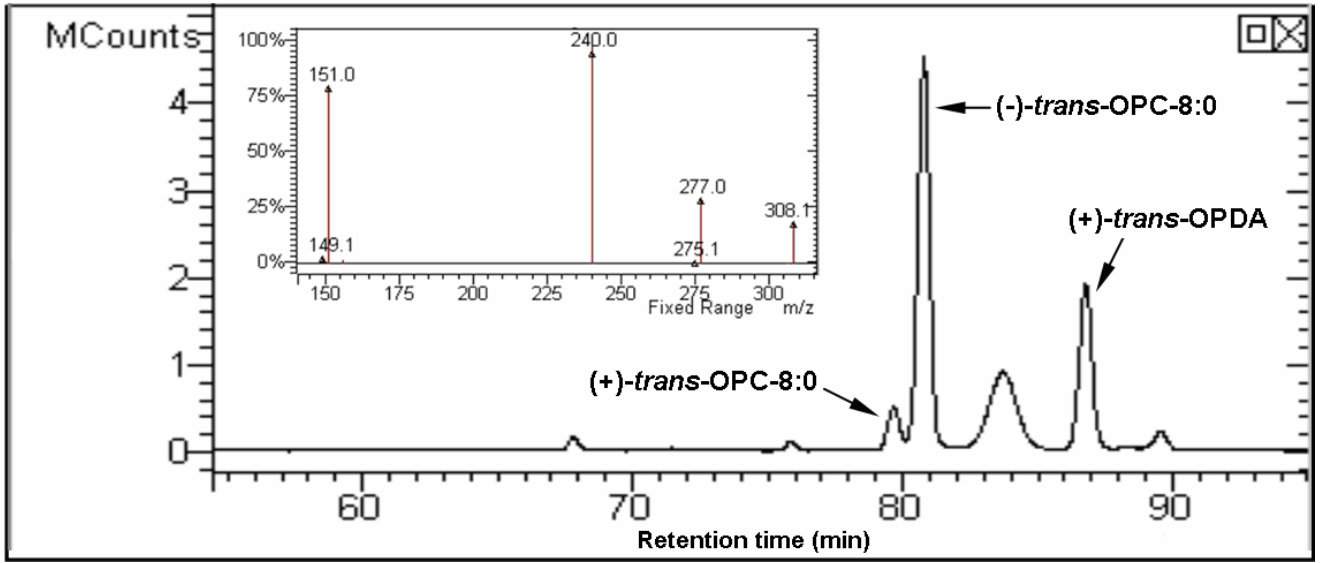

Fig. 4. Chiral GC-MS profile of methylated (+)-trans and (-)-trans-OPC-8:0 from the reaction between coupled recombinant proteins (PpAOS1 and PpOPR1) and 13(S)-HPOT. Inset indicates the mass spectra of methylated OPC-8:0

In contrast, PpOPR3 contains phenylalanine and histidine residues at substrate specificity determining positions (Fig. 3). As the result of that, it should have group II type activity. From the reaction of PpOPR3, a striking result was obtained because PpOPR3 also preferentially reduces the (-)-cis-OPDA into (-)-cis OPC-8:0 behaving as group I OPRs (Fig. $5)$. 


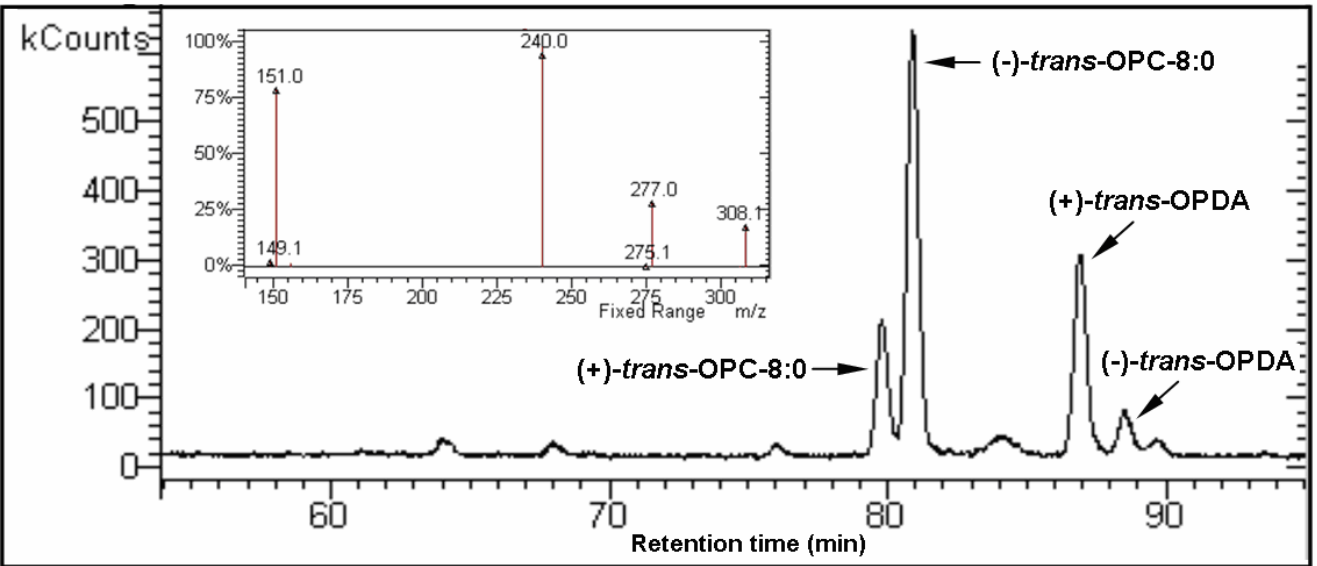

Fig. 5. Chiral GC-MS profile of methylated (+)-trans and (-)-trans-OPC-8:0 from the reaction between combined recombinant proteins (PpAOS1 and PpOPR3) and 13(S)-HPOT. Inset indicates the mass spectra of methylated OPC-8:0

These results clearly indicate that $P$. patens can not reduce (+)-cis OPDA into (+)-cis-OPC8:0 resulting no JA. This unusual functional property of PpOPR3 explains why $P$. patens does not contain JA or its signaling pathway despite the presence of corresponding homologous genes. Based on our results, $(+)$-cis-OPDA generated from first half of the octadecanoid pathway might function as a signaling molecule in $P$. patens instead of JA. It seems that JA signaling pathway might have evolved after the evolutionary split of bryophytes and vascular plants. Moreover, surprising similarities have been found between auxin and JA signaling pathways (mainly their receptors) leading to hypothesize that JA perception mechanism might have evolved from that of auxin (Chico et al., 2008). In addition, auxin signaling pathway is well functioning in P. patens whereas JA signaling pathway is not. Our results also further support this hypothesis.

\section{CONCLUSIONS}

The study shows that it is obvious that $P$. patens cannot biosynthesis JA due to the unusual functional property of PpOPR3. The first half of octadecanoid pathway, which is taking place in the chloroplast, has been conserved in P. patens, and probably in all Bryophytes, to metabolize optically pure $(+)$-cis-OPDA, which might be a signal molecule in $P$. patens instead of JA. Finally, JA signaling pathway seems to have evolved after the evolutionary split of bryophytes and vascular plants.

\section{REFERENCES}

Anterola, A., Gobel, C., Hornung, E., Sellhorn, G., Feussner, I. and Grimes, H. (2009). Physcomitrella patens has lipoxygenases for both eicosanoid and octadecanoid pathways. Phytochem. 70, 40-52.

Breithaupt, C., Kurzbauer, R., Schaller, F., Stintzi, A., Schaller, A., Huber, R., Macheroux, P. and Clausen, T. (2009). Structural basis of substrate specificity of plant 12oxophytodienoate reductases. J. Mol. Biol. 392, 1266-1277. 
Chico, M.J., Chini, A., Fonseca, S. and Solano, R. (2008). JAZ repressors set the rhythm in jasmonate signaling. Curr. Opin. Plant Biol. 11, 486-494.

Chini, A., Fonseca, S., Fernandez, G., Adie, B., Chico, J.M., Lorenzo, O., Casado, G.G., Vidriero, I.L., Lozano, F.M., Ponce, M.R., Micol, J.L. and Solano, R. (2007). The JAZ family of repressors is the missing link in jasmonate signaling. Nature, 448, 666-673.

Cove, D. (2005). The Moss Physcomitrella patens. Annu. Rev. Genet. 39, 339-358.

Fonseca, S., Chini, A., Hamberg, M., Adie, B., Porzel, A., Kramell, R., Miersch, O., Wasternack, C. and Solano, R. (2008). (+)-7-iso-Jasmonoyl-L-isoleucine is the endogenous bioactive jasmonate. Nat.Chem. Biol. 5, 344-350.

Hashimoto, T., Takahashi, K., Sato, M., Bandara, P.K.G.S.S. and Nabeta, K. (2011). Cloning and characterization of an allene oxide cyclase, PpAOC3, in Physcomitrella patens. Plant Growth Regul. 65, 239-245.

Komatsu, K., Nishikawa, Y., Ohtsuka, T., Taji, T., Quatrano, R.S., Tanaka, S. and Sakata, Y. (2009). Functional analyses of the ABI1-related protein phosphatase type $2 \mathrm{C}$ reveal evolutionarily conserved regulation of abscisic acid signaling between Arabidopsis and the moss Physcomitrella patens. Plant Mol. Biol. 70, 327-340.

Muller, J.L., Julke, S., Bierfreund, N.M., Decker, E.L. and Reski, R. (2009). Moss (Physcomitrella patens) GH3 proteins act in auxin homeostasis. New Phytol. 181, 323-338.

Nishiyama, T., Hiwatashi, Y., Sakakibara, K., Kato, M. and Hasebe, M. (2000). Tagged mutagenesis and gene-trap in the moss, Physcomitrella patens by shuttle mutagenesis. DNA Res. 7, 9-17.

Rensing, S.A., Lang, D., Zimmer, A.D. and et al. (2008). The Physcomitrella genome reveals evolutionary insights into the conquest of land by plants. Science, 319, 64-69.

Santner, A. and Estelle, M. (2009). Recent advances and emerging trends in plant hormone signaling. Nature, 459, 1071-1078.

Schaller, F., Schaller, A. and Stintzi, A. (2005). Biosynthesis and metabolism of jasmonates. J. Plant Growth Regul. 23, 179-199.

Schwartzenberg, K.V., Nunez, M.F., Blaschke, H., Dobrev, P.I., Novak, D.O., Motyka, V. and Strnad, M. (2007). Cytokinins in the Bryophyte Physcomitrella patens: analyses of activity, distribution, and cytokinin oxidase/dehydrogenase overexpression reveal the role of extracellular cytokinins. Plant Physiol. 145, 786-800.

Wasternack, C., Miersch, O., Kramell, R., Hause, B., Ward, J., Beale, M., Boland, W., Parthier, B. and Feussner, I. (1998). Jasmonic acid: biosynthesis, signal transduction, gene expression. Fett-Lipid. 100, 139-146. 